(c) 2020, The Authors. Published by FASS Inc. and Elsevier Inc. on behalf of the American Dairy Science Association ${ }^{\circledR}$. This is an open access article under the CC BY-NC-ND license (http://creativecommons.org/licenses/by-nc-nd/4.0/).

\title{
Inoculation with rumen fluid in early life as a strategy to optimize the weaning process in intensive dairy goat systems
}

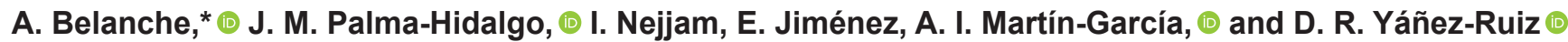 \\ Estación Experimental del Zaidín (CSIC), Profesor Albareda 1, 18008 Granada, Spain
}

\begin{abstract}
Ruminants are born with an undeveloped physical, metabolic, and microbial rumen. Rumen development is limited under artificial rearing systems when newborn animals are separated from the dam, fed on milk replacer, and weaned at an early age. This study aims to evaluate the effects of early-life inoculation of young ruminants with rumen fluid from adult animals. Eighty newborn goat kids were randomly allocated to 1 of 4 experimental treatments and inoculated daily from d 1 to wk 11 with autoclaved rumen fluid (AUT), fresh rumen fluid obtained from adult goats fed either a forage diet (RFF) or concentrate-rich diet (RFC), or absence of inoculation (CTL). Goat kids were artificially reared with ad libitum access to milk replacer, starter concentrate, and forage hay. Blood was sampled weekly and rumen microbial fermentation was monitored at 5 (preweaning), 7 (weaning), and $9 \mathrm{wk}$ of age (postweaning). Results indicated that inoculation with fresh rumen fluid accelerated the rumen microbial and fermentative development before weaning. As a result, RFC and RFF animals had higher solid feed intake $(+73 \%)$, rumen concentrations of ammonia-N $(+26 \%)$, total volatile fatty acids $(+46 \%)$, butyrate $(+50 \%)$, and plasma $\beta$-hydroxybutyrate $(+48 \%)$, and lower milk intake $(-6 \%)$ than CTL and AUT animals at wk 5. Inoculation with fresh inoculum also promoted early rumen colonization by a complex and abundant protozoal community, whereas CTL animals remained protozoa free. Although all kids experienced moderate growth retardation during 1 wk after weaning, inoculation with fresh rumen fluid favored the weaning process, leading to 2.2 times higher weight gain than CTL and AUT animals during wk 8. Some of these advantages were retained during the postweaning period and RFF and RFC animals showed higher forage intake (up to $+44 \%$ ) than CTL and AUT animals with no detrimental effects on feed digestibility or stress levels.
\end{abstract}

Received December 3, 2019.

Accepted February 7, 2020

*Corresponding author: a.belanche@csic.es
The superior microbial load of RFC compared with $\mathrm{RFF}$ inoculum tended to provide further improvements in terms of forage intake, plasma $\beta$-hydroxybutyrate, and rumen protozoa, whereas AUT inoculation provided minor (if any) advantages with respect to CTL animals. Although no differences were noted on animal growth, this study suggests that early life inoculation of goat kids with rumen microbiota can represent an effective strategy to accelerate the rumen development, facilitating a smooth transition from milk to solid feed and to the potential implementation of early weaning strategies.

Key words: artificial rearing, goat kid, microbial inoculation, rumen development, weaning

\section{INTRODUCTION}

Due to increasing global demand for animal-derived food and mounting pressure over land use, there is an urgent need to make livestock production systems more efficient and sustainable (Kim et al., 2019). The viability of any dairy farm depends, to a large extent, on a successful program of rearing newborns for replacement, which implies keeping low mortality rates and feeding costs but assuring an optimal anatomical and functional development to ensure animal performance in adult life (Khan et al., 2016).

Ruminants are born with a physically and metabolically underdeveloped rumen and function as monogastrics over the first weeks of life. Chronologically the rumen development can be divided in 3 phases (Lane et al., 2000): preruminant phase (0-3 wk) in which animals are fed on milk, which bypasses the rumen through the esophageal groove; transition phase (3-8 wk); and ruminant phase (from $8 \mathrm{wk}$ ) in which ruminants are only fed solid diets. Thus, initiation of solid feed consumption, rumen microbial colonization, establishment of rumen fermentation and enzymatic capacity, increment in rumen size, growth and differentiation of papillae, maturation of salivary apparatus, and development of rumination behavior are all needed as the preruminant shifts from milk to solid feed. A smooth transition from a monogastric to ruminant animal is needed to ensure 
a correct anatomical, microbiological, and physiological development to face the weaning nutritional challenge and ultimately to warrant optimal performances later in life (Heinrichs, 2005). This transition generally occurs with no further problems when newborns are reared with the dam, allowing a natural rumen microbial transfer to the offspring (Belanche et al., 2010; Abecia et al., 2014) and feeding behavior learning from the dam (De Paula Vieira et al., 2012). In contrast, in intensive dairy systems newborns are typically separated from their dams after birth and fed either milk replacer or whole milk. This absence of contact with adult animals has been shown to limit the rumen microbial development with negative effects on feed digestibility and productivity (Belanche et al., 2019c). The magnitude of these detrimental effects increases when artificial rearing is combined with early weaning programs to minimize milk-replacer costs, which may lead to weaning-associated shock (Lu and Potchoiba, 1988). Thus, future early-life nutritional strategies should be focused on mimicking the physiological events that occur under natural rearing conditions with the dam.

Among the range of options, probiotics offer a source of microorganisms that could accelerate and modulate the microbial colonization at ruminal and intestinal levels (McAllister et al., 2011). It may be hypothesized that probiotics collected from the rumen may establish more easily into the microbial community than "foreign" species. Several studies have explored the concept of direct-fed microbials in preruminants and adult ruminants (McAllister et al., 2011). Inoculation of adult ruminants with fresh (Rodríguez and Rodríguez, 2011) or lyophilized rumen fluid (Waymack, 1976) has shown minor effects on rumen fermentation and animal performances, which can be explained by the difficulty of modifying a mature and well-established rumen microbial community (Weimer, 2015). In contrast, the developing rumen in the newborn may represent a unique opportunity for the manipulation of rumen microbial colonization (Yáñez-Ruiz et al., 2015). Several authors have evaluated the effects of inoculating young lambs with fresh (Ewan et al., 1958; De Barbieri et al., 2015b) or lyophilized rumen fluid (Abo-Donia et al., 2011; Zhong et al., 2014). Others have evaluated with different degrees of success the effect of inoculating young ruminants with cell-free rumen fluid, bacterial polysaccharides (Muscato et al., 2002), and fermentation products (Górka et al., 2018; O'Hara et al., 2018). The great variability observed across these and other studies suggests that more attention must be given to the selection of the microbial inoculum and to the rearing system because most of these studies were performed under natural milk feeding with the dam, which could minimize the effect of the inoculation. Moreover, to date no studies have been reported using goat kids as experimental animals.

The objective of this study was to optimize the artificial rearing systems of goat kids by implementing new nutritional strategies in early life. It was hypothesized that the inoculation of young goat kids with different types of rumen fluid from adult animals could modify or accelerate the rumen microbial colonization pattern toward a desirable anaerobic fermentation during the preweaning period, facilitate the transition to solid diet postweaning, and increase productivity or decrease feeding costs.

\section{MATERIALS AND METHODS}

\section{Preparation and Characterization of the Rumen Microbial Inocula}

Animal procedures were conducted by trained personnel according to the Spanish guidelines (RD 53/2013) and protocols were approved by the Ethical Committee for Animal Research (EEZ-CSIC) regional government (09/03/2017). Eight Murciano-Granadina goats fitted with permanent rumen cannula were used as rumen fluid donors (mean $\pm \mathrm{SD} ; 46 \pm 4 \mathrm{~kg}$ of BW). Goats were randomly distributed into 2 groups $(\mathrm{n}=4)$ and fed either a forage diet (50\% alfalfa hay and $50 \%$ oat hay) or a concentrate-rich diet $(70 \%$ concentrate and $30 \%$ of the same forage mixture as above). Each group of goats was divided into 2 pairs, which were used as rumen fluid donors on alternate days. Forages were chopped to between 4 and $6 \mathrm{~cm}$ in length by passing through a garden shredder (Bioline 1000, Atika, Ahlen, Germany). The chemical composition of the forage mixture (in DM) was $90.6 \%$ OM, $15.9 \%$ CP, $59.4 \%$ $\mathrm{NDF}, 36.6 \%$ ADF, $9.5 \%$ ADL, and $1.6 \%$ ether extract (EE), whereas the pelleted commercial concentrate (Lactación Rumiantes, Macob, Granada, Spain) was 95.1\% OM, 20.5\% CP, 25.4\% NDF, 7.3\% ADF, $2.1 \%$ $\mathrm{ADL}$, and $4.5 \% \mathrm{EE}$. Diets were offered at 1.2 times maintenance level divided into 2 equal meals $(0800$ and $1600 \mathrm{~h}$ ) and animals were adapted to the diets during 2 wk before being used as donors.

Rumen fluids from donor animals fed forage (RFF) or concentrate diets (RFC) were collected daily at $3 \mathrm{~h}$ after the morning feeding from rumen-cannulated goats fed either the forage diet or a high concentrate-rich diet. A 20-cm-long handle sampling scoop was used to collect rumen contents through the rumen cannula from different parts of the dorsal sac in the rumen (Ramos-Morales et al., 2014). Rumen contents (approximately $100 \mathrm{~mL}$ per animal) were strained through 
Table 1. Inocula characterization for autoclaved rumen fluid (AUT) and rumen fluid from adult goats fed forage (RFF) or concentrate diets (RFC) in terms of rumen fermentation, microbial concentrations by quantitative PCR, and protozoal visual classification

\begin{tabular}{lccccc}
\hline Inoculum & AUT & RFF & RFC & SEM & $P$-value \\
\hline DM, \% & $4.28^{\mathrm{ab}}$ & $2.35^{\mathrm{b}}$ & $5.22^{\mathrm{a}}$ & 0.620 & 0.043 \\
$\mathrm{pH}$ & $6.11^{\mathrm{b}}$ & $6.38^{\mathrm{a}}$ & $5.79^{\mathrm{c}}$ & 0.067 & 0.002 \\
Lactate, mM & $1.86^{\mathrm{a}}$ & $0.55^{\mathrm{b}}$ & $0.85^{\mathrm{b}}$ & 0.232 & 0.017 \\
Ammonia-N, mg/dL & 8.64 & 7.27 & 10.0 & 0.774 & 0.117 \\
Total VFA, mM & $120^{\mathrm{ab}}$ & $103^{\mathrm{b}}$ & $134^{\mathrm{a}}$ & 7.064 & 0.055 \\
Acetate, \% & $63.0^{\mathrm{b}}$ & $70.1^{\mathrm{a}}$ & $55.5^{\mathrm{c}}$ & 0.947 & $<0.001$ \\
Propionate, \% & $23.6^{\mathrm{b}}$ & $18.2^{\mathrm{c}}$ & $29.0^{\mathrm{a}}$ & 1.210 & $<0.001$ \\
Butyrate, \% & $10.3^{\mathrm{b}}$ & $9.49^{\mathrm{c}}$ & $11.8^{\mathrm{a}}$ & 0.527 & 0.004 \\
Microbiota, log copies/mL $_{\text {Bacteria }}$ & - & & & & \\
Methanogens & - & 9.96 & 11.8 & 0.625 & 0.081 \\
Anaerobic fungi & - & 6.32 & 7.65 & 0.858 & 0.314 \\
Protozoa & - & $6.66^{\mathrm{b}}$ & $7.55^{\mathrm{a}}$ & 0.088 & $<0.001$ \\
Main protozoal group & - & & & 0.349 & 0.005 \\
$\quad$ Subfamily Entodiniinae, \% & - & 80.2 & 83.3 & 4.490 & 0.646 \\
Subfamily Diplodiniinae, \% & - & 6.72 & 5.30 & 1.853 & 0.612 \\
Ophryoscolex spp., \% & - & 0.32 & 1.85 & 0.785 & 0.226 \\
Isotricha spp., \% & - & 2.33 & 1.41 & 0.993 & 0.542 \\
Dasytricha sp., \% & - & 10.4 & 8.12 & 2.8072 & 0.587 \\
\hline
\end{tabular}

${ }^{\mathrm{a}-\mathrm{c}}$ Means within a row with different superscripts differ $(P<0.05)$.

double cheesecloth, bubbled with $\mathrm{CO}_{2}$, pooled by diet, maintained at $37^{\circ} \mathrm{C}$ in a prewarmed thermal flask, and immediately administered as inoculum to young kids. Autoclaved inoculum (AUT) was prepared weekly by mixing equal volumes of RFF and RFC inocula from all donors and autoclaved at $115^{\circ} \mathrm{C}$ for 30 min to destroy all viable microbes while maintaining fermentation products. A subsample from each pooled inocula was taken every 2 wk (resulting on 4 samples per inocula) to describe their composition in terms of percentage of DM, rumen fermentation ( $\mathrm{pH}, \mathrm{VFA}$, ammonia, and lactate), and abundance of the main microbial groups by quantitative PCR (qPCR; Table 1).

\section{Inoculation Experiment}

A total of 80 newborn Murciano-Granadina goat kids born within a 2-wk period were used. At birth animals were weighed, separated from their mothers, and fed with natural colostrum via esophageal probe (approximately $200 \mathrm{~mL}$ divided in 2 doses). Animals were randomly allocated to 1 of 4 experimental treatments. During this allocation process, sex and initial BW were considered, resulting in similar sex distribution and initial BW across treatments. Although the mother effect was not considered, siblings were always allocated to different treatments. Treatment consisted of oral inoculation from d 1 to wk 11 of age with AUT, $\mathrm{RFF}, \mathrm{RFC}$, or absence of inoculation (CTL). Inoculation was conducted daily using a syringe connected to a $10-\mathrm{cm}$ tube to drench the inocula $(2.5 \mathrm{~mL} /$ animal during wk 1 and $5 \mathrm{~mL} /$ animal thereafter), making sure that all volume was swallowed.

Animals from different treatments were physically separated by a $2-m$-wide corridor to prevent physical contact. To monitor feed intake, animals within each treatment were distributed in 5 contiguous pens with an equal number of males and females. All 4 animals within the same pen number ( 1 to 5 according to birth order) had similar age (maximum $2 \mathrm{~d}$ difference) and were handled and sampled on the same day across treatments. All animals were raised on milk replacer (declared composition in DM: $92.8 \%$ OM, $24.0 \% \mathrm{CP}$, and $22.0 \% \mathrm{EE}$ ), which was offered ad libitum and freshly prepared twice per day (Univet Spray, Cargill, Barcelona, Spain). From wk 2, animals had free access to the same forage mixture that has been described for the donor goats and to pelleted starter concentrate (0-14 Rumiantes Transición, Macob, Granada, Spain) with $3 \mathrm{~mm}$ diameter. The starter had the following chemical composition (in DM): $94.9 \%$ OM, $22.6 \% \mathrm{CP}$, $3.19 \%$ NDF, $8.72 \%$ ADF, 3.38\% ADL, $4.83 \% \mathrm{EE}$; and ingredient list: wheat bran, corn, sunflower seeds, barley, wheat, soybean flour, $\mathrm{CaCO}_{3}, \mathrm{NaCl}$, and vitaminmineral premix. Animals were weaned at 7 wk of age by progressively decreasing milk powder concentration for $4 \mathrm{~d}$. Intakes were measured daily in each pen and BW was recorded weekly. Feed efficiency was calculated as a ratio between the ADG and ME intake based on their declared composition and the Fundación Española para el Desarrollo de la Nutrición Animal feed tables (de Blas et al., 2010). 


\section{Sampling and Analysis}

Blood samples $(4 \mathrm{~mL})$ were collected at $0900 \mathrm{~h}$ from the jugular vein at $1,3,5,7,9$, and 11 wk of age and placed in tubes with anticoagulant $\left(\mathrm{K}_{3}\right.$-EDTA). Blood was centrifuged at $2,000 \times g$ for $15 \mathrm{~min}$ at room temperature and plasma was collected to determine glucose and BHB levels using an auto-analyzer (BA400, BioSystems, Barcelona, Spain). Rumen microbial fermentation was studied at wk 5 (preweaning), 7 (weaning), and 9 (postweaning). Rumen content was withdrawn from each animal by orogastric intubation at $0900 \mathrm{~h}$ as previously described (Ramos-Morales et al., 2014): a flexible polyvinyl chloride tube $(8 \mathrm{~mm}$ internal diameter) with about 20 holes of $5 \mathrm{~mm}$ diameter in the $12-\mathrm{cm}$ probe head was warmed up with hot water, externally lubricated with sunflower oil, and inserted to a depth of approximately $60 \mathrm{~cm}$ via the esophagus. Rumen samples (approximately $50 \mathrm{~mL}$ ) were obtained using an electric vacuum pump (down to 9 mbar; Vacuubrand MZ 2C, Wertheim, Germany) connected to a sterile collection container. Samples were filtrated through cheesecloth and solids were discarded given the small and variable proportion of solids in the samples. Then, $\mathrm{pH}$ was measured and 4 subsamples were taken for VFA, ammonia, lactate, and protozoal optical counting, respectively (Belanche et al., 2019c). During the postweaning period (10 wk of age), fecal samples were collected from each animal during 3 consecutive days, which together with feed offered and orts were used to determine feed digestibility using the $\mathrm{Mn}$ as internal marker (Hidiroglou, 1979). For feed, orts, and feces, chemical composition was determined as previously described (Arco-Pérez et al., 2017), whereas Mn was measured by optical-ICP spectrometry (720-ES ICPOES spectrometer, Agilent Technologies, Santa Clara, CA). Rumen concentrations of individual VFA were determined by a GC system coupled with a Flame Ionization Detector (Auto-System, Perkin Elmer, Waltham, MA), whereas ammonia concentration was measured using a colorimetric method (Weatherburn, 1967). Protozoal concentration and classification were visually determined (Dehority, 1993) using a Sedgewick rafter counting chamber and an optical microscope (Nikon Labophot, Tokyo, Japan). For inocula characterization, freeze-dried samples were bead-beated for $1 \mathrm{~min}$ (MiniBeadBeater, Biospect Products, Bartlesville, OK) and DNA was extracted using a commercial kit (QIAamp DNA Stool Mini Kit, Qiagen Ltd., Barcelona, Spain). Concentrations of total bacteria, methanogenic archaea, protozoa, and anaerobic fungi were determined by quantitative PCR as previously described (Belanche et al., 2016). Primer sets used were as follows: $16 \mathrm{~S}$ rRNA forward GTGSTGCAYGGYTGTCGTCA and reverse
ACGTCRTCCMCACCTTCCTC for total bacteria; the mcrA gene forward TTCGGTGGATCDCARAGRGC and reverse GBARGTCGWAWCCGTAGAATCC for methanogenic archaea; the 18S rRNA forward GAGGAAGTAAAAGTCGTAACAAGGTTTC and reverse CAAATTCACAAAGGGTAGGATGAT for anaerobic fungi; and the 18S rRNA forward GCTTTCGWTGGTAGTGTATT and reverse CTTGCCCTCYAATCGTWCT for protozoa. Cycling conditions were $95^{\circ} \mathrm{C}$ for $5 \mathrm{~min}$; 40 cycles of $95^{\circ} \mathrm{C}$ for $15 \mathrm{~s}, 60^{\circ} \mathrm{C}$ for $30 \mathrm{~s}$, and $72^{\circ} \mathrm{C}$ for $55 \mathrm{~s}$; and $72^{\circ} \mathrm{C}$ for $1 \mathrm{~min}$. The absolute amount of each microbial group, expressed as DNA copies $/ \mathrm{mL}$ of fresh matter, was determined using serial dilutions of known amounts of standards. The qPCR standards consisted of the plasmid pCR 4-TOPO (Invitrogen, Carlsbad, CA) with inserted 16S, mcrA, or $18 \mathrm{~S}$ gene fragments from each microbial group, respectively.

To assess the potential effect of the experimental treatments on postweaning stress, cortisol concentration in hair was measured (Moya et al., 2013). Briefly, a surface of $25 \mathrm{~cm}^{2}$ in the dorsal neck was shaved at weaning (wk 7) and the hair grown during the postweaning was collected at wk 10 . Hair samples $(250 \mathrm{mg})$ were washed twice for $3 \mathrm{~min}$ with isopropanol, dried, and bead-beated 3 times for 2 min at maximum speed. Hair samples were sonicated $30 \mathrm{~min}$ and incubated with isopropanol $18 \mathrm{~h}$ at $50^{\circ} \mathrm{C}$ with constant mixing. Supernatant $(0.8 \mathrm{~mL})$ was extracted, evaporated, reconstituted in PBS $(0.2 \mathrm{~mL})$, and cortisol concentration was measured using a commercial kit (Cortisol ELISA Saliva, ALPCO, Salem, NH).

\section{Calculation and Statistical Analyses}

Two animals per treatment were removed from the study due to deaths and health problems over the course of the study. Protozoa optical counting and qPCR data were $\log _{10}$ transformed to attain normal distributions. Rumen protozoa and fermentation data were analyzed based on a repeated measures mixed-effects model (residual maximum likelihood) as follows:

$$
Y_{i j k l}=\mu+I_{i}+T_{j}+(I \times T)_{i j}+G_{k}+A(G)_{l}+e_{i j k l},
$$

where $Y_{i j k l}$ is the dependent, continuous variable, $\mu$ is the overall population of the mean, $I_{i}$ is the fixed effect of the inoculation ( $i=\mathrm{CTL}$ vs. AUT vs. RFF vs. RFC), $T_{j}$ is the fixed effect of the sampling time or age $(j=5$ vs. 7 vs. 9 wk $),(I \times T)_{i j}$ is the interaction term, $G_{k}$ is the random effect of the pen considered as a block ( $k=1$ to 5 ), $A(G)_{l}$ is the random effect of the animal nested to the pen $(l=1$ to 80$)$, and $e_{i j k l}$ is the residual error. For BW and ADG data, the sex was 
also included as a fixed factor. The pen was considered as the experimental unit for feed intake and feed efficiency. When significant effects were detected, means were compared by Fisher's protected least significant difference test using SPSS software (version 21.0, IBM Corp., New York, NY). Significant effects were declared at $P<0.05$ and tendency to difference at $P<0.1$.

\section{RESULTS}

\section{Description of the Inocula}

Substantial differences were detected across the 3 rumen inocula (Table 1), with RFC inoculum being higher in concentrations of DM, lactate, total VFA, propionate, butyrate, bacteria, anaerobic fungi, and protozoa than RFF inoculum. On the contrary, RFF inoculum had higher $\mathrm{pH}$ and acetate molar proportion than RFC inoculum. No differences were noted between RFC and RFF inocula on the methanogen concentrations determined by $\mathrm{qPCR}$ and on the protozoal group distribution after visual analysis. Autoclaved rumen fluid had intermediate rumen fermentation values in comparison to RFF and RFC given that AUT was generated by mixing equal volumes of both inocula. Autoclaved inocula showed high lactate concentration and undetectable concentrations of bacteria, methanogens, and fungal DNA, as well as the complete disruption of rumen protozoa since no whole protozoal cells were evident after visual inspection.

\section{Feed Intake, Digestibility, and Blood Metabolites}

Starter feed and forage intakes remained low until weaning and increased exponentially during the postweaning period (Figure 1), but with differences for forage intake across treatments as revealed by the interaction between inoculation and time $(P<0.001)$. Inoculation of young animals with fresh rumen fluid (RFF and RFC) promoted higher concentrate intake (Figure 1A) during the preweaning period (wk 3, 4, 5 , and 6), as well as higher forage intake (Figure 1B) during the postweaning period (wk 5, 9, 10, and 11). On the contrary, animals in treatments CTL and AUT tended to have a higher milk replacer intake (1.35 vs. $1.27 \mathrm{~L} / \mathrm{d}, P=0.096)$.

The analysis of the blood metabolites (Figure 1) showed a progressive decline of the plasma glucose $(P<$ $0.001)$ and an increase of the BHB concentration as the trial progressed $(P<0.001)$. However, the concentration of BHB showed a significant interaction between inoculation and time $(P<0.001)$, indicating that animals inoculated with fresh rumen fluid had increasingly higher BHB levels at wk $5(P=0.052), 7(P=0.027)$,
$9(P=0.004)$, and $11(P<0.001)$ in comparison to CTL and AUT animals. On the contrary, inoculation with fresh rumen fluid tended to lower blood glucose concentration at wk $7(P=0.10)$ and $9(P=0.012)$.

To further investigate the effect of the inoculation on the transition from liquid to solid diet, the feed digestibility and cortisol level in hair were measured 3 wk after weaning (Table 2). No differences in total DMI were noted at wk 10 across treatments despite diets being offered ad libitum; however, the forage intake represented a higher proportion of the total DMI in animals inoculated with fresh rumen fluid (mostly RFC) than in CTL animals $(P=0.012)$. Despite these differences in the forage-to-concentrate ratio across treatments, no significant differences in total-tract apparent digestibility were noted for OM, N, NDF, and ADF. The analysis of the cortisol level in hair showed no significant differences across treatments.

\section{Rumen Protozoa and Microbial Fermentation}

Control animals remained protozoa free throughout the entire duration of the study (Table 3), whereas for the rest of the treatments a progressive increase in protozoal concentration occurred over time $(P<0.001)$ with increasing numbers of Isotricha spp., Dasytricha sp., and Entodiniinae to the detriment of subfamily Diplodiniinae. The significant interaction between inoculation and time observed for the protozoal concentration $(P=$ 0.025 ) indicated that inoculation of young animals with fresh rumen fluid promoted early rumen colonization by an abundant and diverse protozoal population. As a result RFF and RFC had higher protozoal concentration than AUT goat kids across times, with the highest concentration being observed in RFC animals at $9 \mathrm{wk}$ of age. For the AUT treatment, the protozoal community was mostly conformed by subfamily Entodiniinae (average 98\% across sampling times) and no holotrich protozoa were detected in these animals throughout the experiment. On the contrary, RFF and RFC animals had a lower abundance of Entodiniinae (average $79 \%$ across sampling times) and higher abundances of other protozoal groups such as Diplodiniinae (9.2\%), Ophryoscolex spp. (4.7\%), Isotricha spp. (2.3\%), and Dasytricha sp. (4.5\%) in comparison to AUT animals.

An escalation in the rumen fermentative activity was observed over time as noted by increasing values for total VFA, propionate, butyrate, and valerate along with decreasing values for ammonia, acetate, isobutyrate, and isovalerate molar proportions (Table $4, P<0.001$ ). The significant interaction between inoculation and time observed in most fermentation variables indicated that the inoculation with fresh rumen fluid accelerated the rumen fermentative development. As a result, at 
5 wk of age RFF and RFC animals had higher total VFA concentration than CTL and AUT animals (33.9 vs. $23.1 \mathrm{~m} M, P<0.001)$; these figures are similar to those observed at wk 7 across treatments $(33.1 \mathrm{mM})$. During the preweaning period, RFF animals had the highest ammonia- $\mathrm{N}(P=0.028)$ and butyrate levels $(P$ $=0.002)$, whereas AUT animals had the highest isobutyrate $(P<0.001)$ and isovalerate levels $(P=0.001)$. At weaning (wk 7), AUT animals had the highest levels of valerate and isovalerate across treatments. The larg-

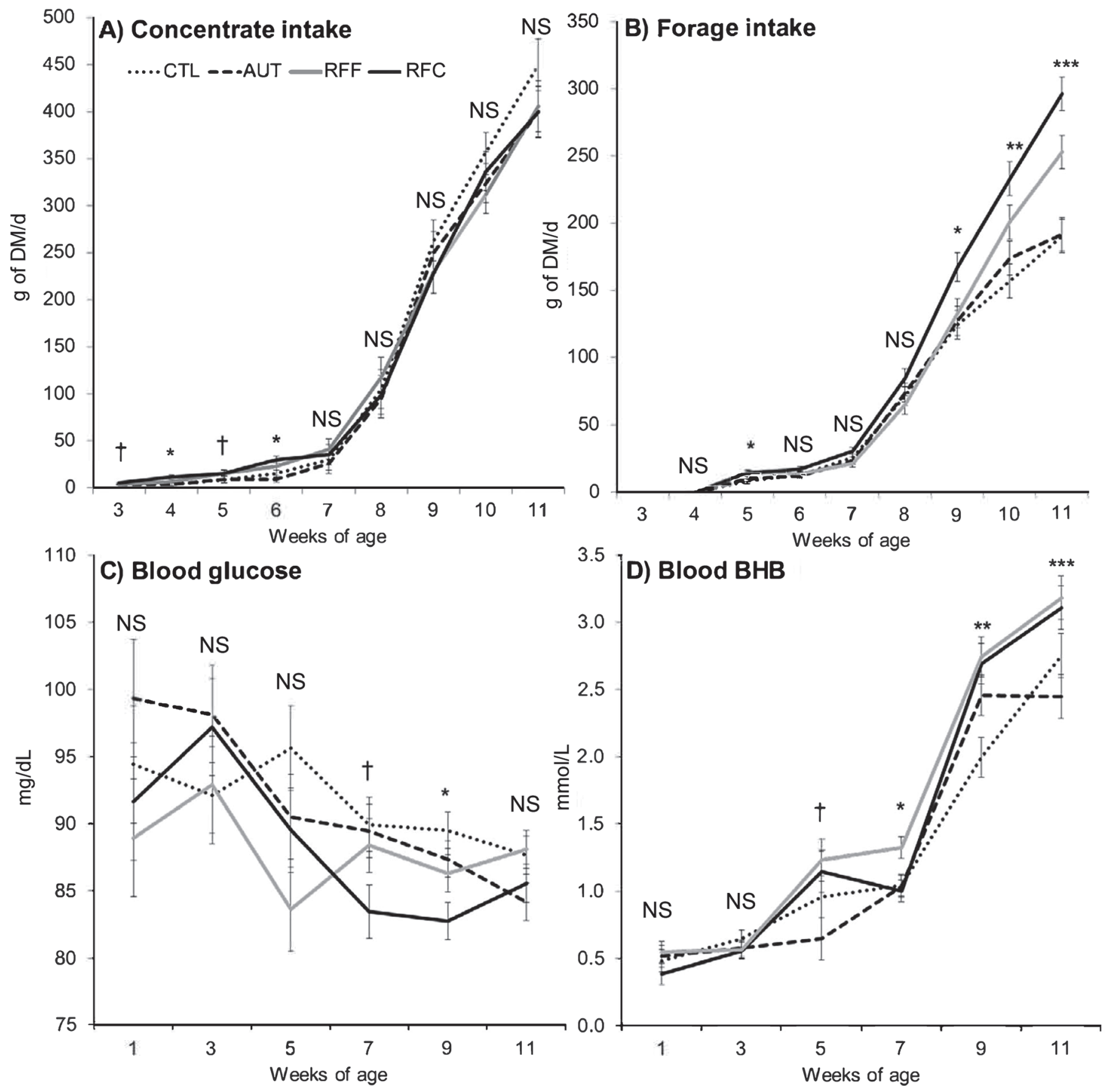

Figure 1. Weekly progression of concentrate intake (A), forage intake (B) and blood concentration of glucose (C) and BHB (D) in goat kids inoculated with autoclaved rumen fluid (AUT, dashed line), rumen fluid from adult animals fed forage (RFF, gray solid line), rumen fluid from adult animals fed forage concentrate diets (RFC, black solid line), or without inoculation (CTL, dotted line). ${ }^{* * *} P<0.001,{ }^{* *} P<0.01,{ }^{*} P<$ $0.05, \dagger P<0.1$, NS: $P>0.1$. Significant interactions between inoculation and time were noted for forage intake and BHB $(P<0.001)$. Error bars indicate SE of the difference. 
Table 2. Intake and feed digestibility during postweaning (wk 10) in goats inoculated with autoclaved rumen fluid (AUT), rumen fluid from adult animals fed forage (RFF) or concentrate diets (RFC), and without inoculation (CTL)

\begin{tabular}{lcccccc}
\hline Item & CTL & AUT & RFF & RFC & SEM & $P$-value \\
\hline DMI, kg/d & 573 & 549 & 582 & 628 & 30.90 & 0.131 \\
Forage, \% of DMI & $32.0^{\mathrm{c}}$ & $35.7^{\mathrm{bc}}$ & $40.3^{\mathrm{ab}}$ & $45.5^{\mathrm{a}}$ & 2.468 & 0.012 \\
Digestibility, \% & & & & & & \\
DM & 78.4 & 76.6 & 78.5 & 75.2 & 0.011 & 0.151 \\
OM & 79.7 & 78.2 & 80.1 & 77.0 & 0.011 & 0.179 \\
$\mathrm{~N}$ & 76.8 & 74.5 & 79.1 & 76.5 & 0.013 & 0.132 \\
NDF & 59.2 & 57.3 & 61.9 & 55.8 & 0.023 & 0.307 \\
ADF & 60.1 & 56.0 & 59.4 & 53.1 & 0.033 & 0.431 \\
Cortisol in hair, ng/mg & 1.17 & 1.12 & 1.19 & 1.16 & 0.063 & 0.878 \\
\hline
\end{tabular}

${ }^{\mathrm{a}-\mathrm{c}}$ Means within a row with different superscripts differ $(P<0.05)$.

est differences in rumen microbial fermentation profiles were detected during the postweaning period (wk 9) as RFF and RFC animals showed higher butyrate and isobutyrate molar proportions and lower total VFA and propionate levels than AUT and CTL animals.

\section{Animal Performance and Feeding Costs}

The animals' sex had a significant effect on ADG (Table 5) as males showed higher BW than females from wk 7, as well as higher ADG from wk 3 (data not shown). No differences were noted in the BW and ADG across treatments during the entire duration of this study; however, for ADG there was a significant interaction between inoculation and time $(P=0.004)$. This interaction suggested that although the weaning process led to an important decrease in the ADG during the first week after weaning (wk 8), the inoculation with fresh rumen fluid helped to ameliorate this decrease, leading to higher ADG values than observed in AUT and CTL animals. Given the different ME density among milk replacer, concentrate, and forage, the feed efficiency was calculated based on total ME intake (Table 5). Feed efficiency decreased over time, but this decrease was modulated by the inoculation as an interaction was noted $(P=0003)$. This interaction suggested that animals inoculated with fresh rumen fluid tended to have higher feed efficiency than CTL

Table 3. Concentration of the main protozoa groups assessed by optical microscopy in goats inoculated with autoclaved rumen fluid (AUT), rumen fluid from adult animals fed forage (RFF) or concentrate diets (RFC), and without inoculation (CTL)

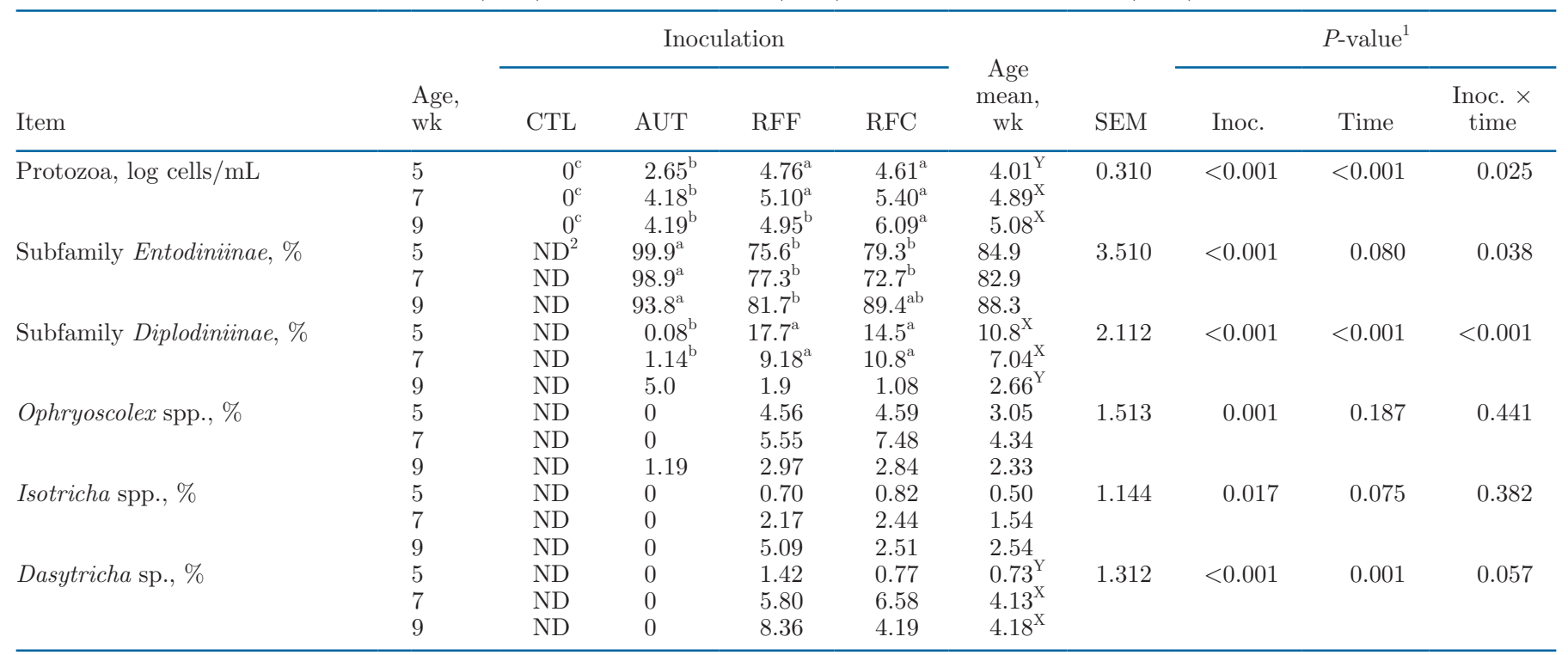

\footnotetext{
${ }^{\mathrm{a}-\mathrm{c}}$ Means within a row with different superscripts differ $(P<0.05)$.

${ }^{\mathrm{X}, \mathrm{Y}}$ Means within a column with different superscripts differ $(P<0.05)$.

${ }^{1}$ Inoc. $=$ inoculation

${ }^{2} \mathrm{ND}=$ not detected.
} 
and AUT animals during the following week to weaning (63.5 vs. $17.3 \mathrm{~kg} /$ Mcal of ME).

The accumulated feed consumption and feeding costs were calculated over the duration of this study (11 wk) to assess the economic feasibility of these interventions (Table 6). During the milk feeding period, RFC animals (followed by RFF) tended to have lower milk intake than CTL and AUT animals (10.1 vs. $11.0 \mathrm{~kg}$ of milk powder/animal). No differences were noted on the concentrate intake across treatments $(8.83 \pm 0.68 \mathrm{~kg}$ of $\mathrm{DM}$ /animal), whereas RFC (followed by RFF animals) had substantially higher forage intake than CTL and AUT animals (6.35 vs. $4.52 \mathrm{~kg}$ of $\mathrm{DM} /$ animal). The total feeding cost up to wk 11 was calculated taking in consideration the different purchased feed costs [2.24, 0.34 , and $0.17 € / \mathrm{kg}$ ( $1 €$ is approximately equal to USD $\$ 1.15$ ) for milk replacer, concentrate, and forage, respectively]. Animals within the RFC treatment tended to have lower milk replacer costs than CTL and AUT animals $(P=0.096)$, whereas the opposite was true for forage $\operatorname{costs}(P=0.001)$, and no differences were noted for the concentrate feeding cost across animals. As a result, no differences were noted across treatments on the overall feeding cost per animal or per kilogram of BW gained.

\section{DISCUSSION}

\section{Selection and Description of the Inocula}

In a previous publication we evaluated the microbial activity of different types of rumen inocula, sampling times, and preservation methods, concluding that fresh rumen fluid sampled at $3 \mathrm{~h}$ after feeding provides the most diverse and active inoculum based on in vitro incubations (Belanche et al., 2019b). The present in vivo study builds upon this previous observation and indicated that RFC inoculum had higher concentration of total bacteria, anaerobic fungi, protozoa, VFA, propionate, and butyrate than RFF inoculum. This superior microbial activity for RFC than for RFF inocula has also been demonstrated in vitro (higher VFA, ammonia, and gas production) after having been incubated with the same substrate (Belanche et al., 2019b). Although the microbes in the inocula were not taxonomically characterized, the different VFA profile

Table 4. Rumen microbial fermentation in goats inoculated with autoclaved rumen fluid (AUT), rumen fluid from adult animals fed forage (RFF) or concentrate diets (RFC), and without inoculation (CTL)

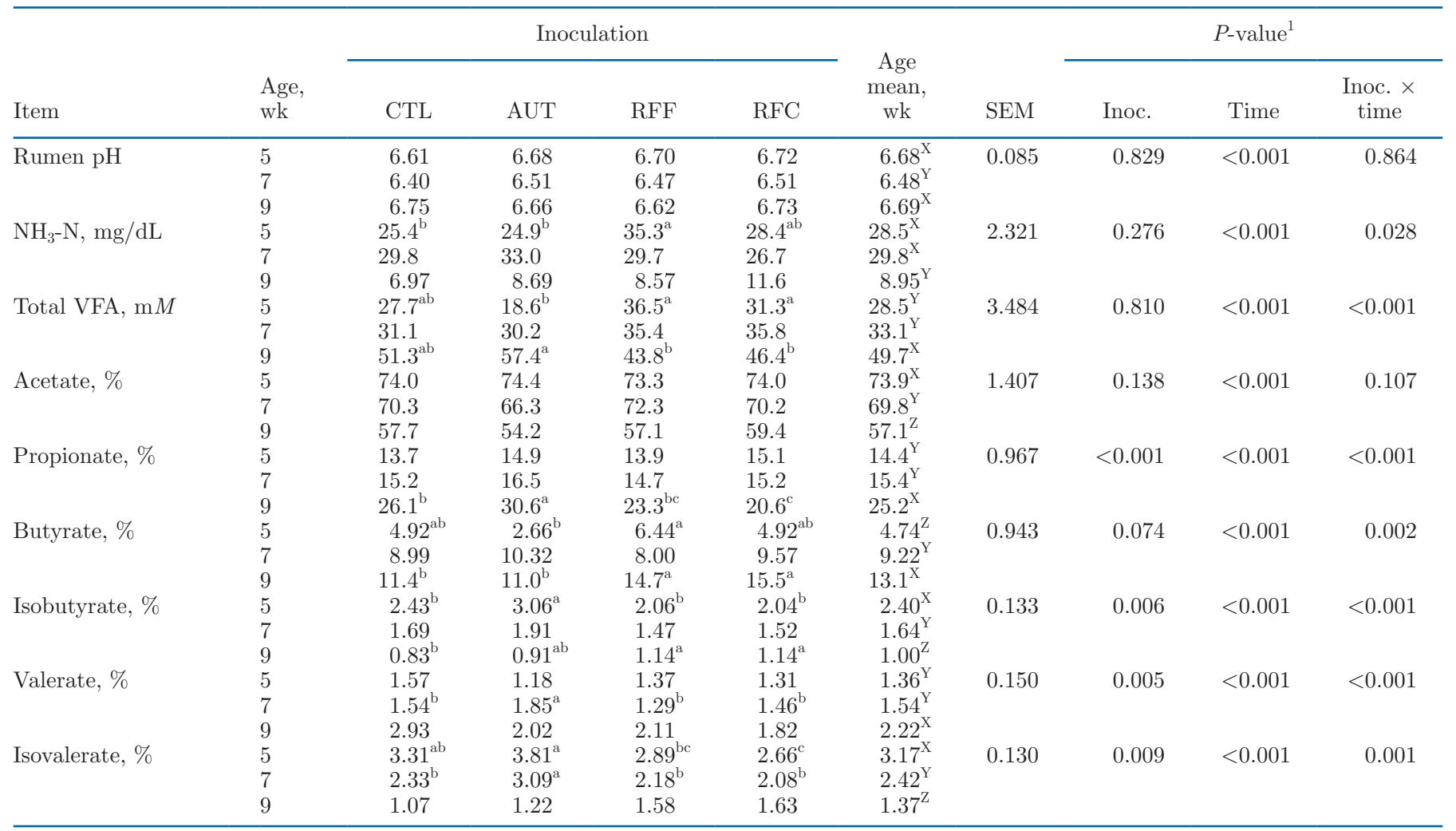

${ }^{\mathrm{a}-\mathrm{c}}$ Means within a row with different superscripts differ $(P<0.05)$.

${ }^{\mathrm{X}-\mathrm{Z}}$ Within a column means with different superscripts differ $(P<0.05)$.

${ }^{1}$ Inoc. $=$ inoculation. 
Table 5. Body weight, ADG, and feed efficiency (FE) in goats inoculated with autoclaved rumen fluid (AUT), rumen fluid from adult animals fed forage (RFF) or concentrate diets (RFC), and without inoculation (CTL)

\begin{tabular}{|c|c|c|c|c|c|c|c|c|c|}
\hline \multirow[b]{2}{*}{ Item } & \multirow[b]{2}{*}{$\begin{array}{l}\text { Age, } \\
\text { wk }\end{array}$} & \multicolumn{4}{|c|}{ Inoculation } & \multirow[b]{2}{*}{ SEM } & \multicolumn{3}{|c|}{$P$-value ${ }^{1}$} \\
\hline & & CTL & $\mathrm{AUT}$ & $\mathrm{RFF}$ & $\mathrm{RFC}$ & & Inoc. & Time & $\begin{array}{c}\text { Inoc. } \times \\
\text { time }\end{array}$ \\
\hline \multirow{4}{*}{$\overline{\mathrm{BW}}, \mathrm{kg}$} & 0 & 2.65 & 2.72 & 2.78 & 2.69 & \multirow{4}{*}{0.245} & \multirow[t]{4}{*}{0.737} & \multirow[t]{4}{*}{$<0.001$} & \multirow[t]{4}{*}{0.861} \\
\hline & 3 & 5.31 & 5.58 & 5.13 & 5.10 & & & & \\
\hline & 5 & 6.43 & 6.62 & 6.25 & 6.13 & & & & \\
\hline & 7 & 8.81 & 8.96 & 8.62 & 8.38 & & & & \\
\hline \multirow[t]{6}{*}{$\mathrm{ADG},{ }^{1} \mathrm{~g} / \mathrm{d}$} & $0-3$ & 126 & 136 & 112 & 115 & \multirow[t]{6}{*}{0.011} & \multirow[t]{6}{*}{0.842} & \multirow[t]{6}{*}{$<0.001$} & \multirow[t]{6}{*}{0.004} \\
\hline & $3-5$ & 156 & 150 & 157 & 146 & & & & \\
\hline & $5-7$ & 184 & 184 & 185 & 174 & & & & \\
\hline & 8 & $48.7^{\mathrm{bc}}$ & $27.9^{\mathrm{c}}$ & $75.4^{\mathrm{ab}}$ & $97.1^{\mathrm{a}}$ & & & & \\
\hline & 9 & 178 & 177 & 154 & 165 & & & & \\
\hline & $9-11$ & 173 & 160 & 172 & 181 & & & & \\
\hline $\mathrm{FE}, \mathrm{kg} / \mathrm{Mcal}$ of $\mathrm{ME}$ & $0-3$ & 234 & 240 & 214 & 220 & 10.40 & 0.859 & $<0.001$ & 0.003 \\
\hline
\end{tabular}

${ }^{a-c}$ Means within a row with different superscripts differ $(P<0.05)$.

${ }^{1}$ Inoc. $=$ inoculation.

suggests the presence of a different microbial community (Li et al., 2019).

Within the group of probiotics targeting the rumen (e.g., Saccharomyces, Aspergillus, Megasphaera elsdenii, and Prevotella bryantii), various modes of action have been suggested including stabilization of ruminal $\mathrm{pH}$, oxygen scavenging, and microbial modulation (Yoon and Stern, 1995). However, there is still controversy whether similar effects could be achieved inoculating microbial extracts (Muscato et al., 2002; Uyeno et al., 2015) or fermentation products such as lactate or butyrate (Górka et al., 2018). To explore this hypothesis, autoclaved rumen fluid was used as inoculum because it would provide a comparable amount of fermentation products to fresh rumen fluid (including VFA, ammonia, peptides, and microbial polysaccharides), but without viable cells, as noted by the undetectable concentration of microbial DNA and intact protozoal cells after optical inspection.

\section{Effect of Inoculation Before Weaning}

During the preruminant phase $(0-3 \mathrm{wk})$ there is a substantial increase in the microbial mass in the rumen, this increment being higher in animals with natural rather than artificial rearing (Abecia et al., 2014). However, the inoculation with fresh rumen fluid accelerated the transition phase from liquid to solid diet $(3-8 \mathrm{wk})$, resulting in $68 \%$ higher concentrate intake and $12 \%$ higher forage intake than CTL and AUT animals. Nevertheless, CTL and AUT animals compensated this situation with a slightly higher milk replacer

Table 6. Cumulative feed intake during $11 \mathrm{wk}(\mathrm{DM})$ and feeding costs in goats inoculated with autoclaved rumen fluid (AUT), rumen fluid from adult animals fed forage (RFF) or concentrate diets (RFC), and without inoculation (CTL)

\begin{tabular}{lcccccc}
\hline Treatment & CTL & AUT & RFF & RFC & SEM & $P$-value \\
\hline Milk powder intake, kg/animal & 11.0 & 11.1 & 10.7 & 10.1 & 0.271 & 0.096 \\
Concentrate intake, kg/animal & 9.34 & 8.47 & 8.73 & 8.77 & 0.677 & 0.830 \\
Forage intake, kg/animal & $4.44^{\mathrm{c}}$ & $4.61^{\mathrm{bc}}$ & $5.27^{\mathrm{b}}$ & $6.35^{\mathrm{a}}$ & 0.267 & 0.001 \\
Feeding cost, $€$ & & & & & \\
Milk replacer & $24.5^{\mathrm{a}}$ & $24.9^{\mathrm{a}}$ & $23.9^{\mathrm{ab}}$ & $22.6^{\mathrm{b}}$ & 0.606 & 0.096 \\
Concentrate & 3.18 & 2.88 & 2.97 & 2.98 & 0.231 & 0.830 \\
Forage & $0.76^{\mathrm{c}}$ & $0.78^{\mathrm{bc}}$ & $0.90^{\mathrm{b}}$ & $1.08^{\mathrm{a}}$ & 0.045 & 0.001 \\
Total cost & 28.5 & 28.5 & 27.7 & 26.7 & 0.709 & 0.276 \\
Cost/gain, $€ / \mathrm{kg}$ & 2.50 & 2.54 & 2.48 & 2.40 & 0.060 & 0.435 \\
\hline
\end{tabular}

${ }^{\mathrm{a}-\mathrm{c}}$ Means within a row with different superscripts differ $(P<0.05)$. 
intake $(+6.3 \%)$, resulting in similar ME intake across treatments. Several works have indicated that during this transition phase the presence of solid feed in the rumen (preferably as forage) exerts a physical stimuli favoring rumen anatomical development (Beharka et al., 1998), whereas high milk intake favors BW gain but does not prepare the rumen for successful weaning (Meale et al., 2016).

Whereas rumen bacteria and methanogens are early rumen colonizers (Abecia et al., 2014), protozoa colonize the rumen later because they are highly sensitive to oxygen and require direct contact between young and adult animals for an effective transmission (Bird et al., 2010). As a result, CTL animals remained protozoa free during the entire duration of the study, as we have previously described in artificially reared lambs (Belanche et al., 2019c). A natural sequence of rumen colonization has been described for the different protozoal families starting with Entodiniinae, followed by Diplodiniinae and Ophryoxcolex spp. and finishing with holotrichs (Williams and Coleman, 1992). The visual microscopy examination of the protozoal community confirmed this colonization sequence and showed that inoculation with fresh rumen fluid accelerated this process. The presence of a small protozoal concentration (2.65 $\log _{10}$ cells $\left./ \mathrm{mL}\right)$, mostly composed of Entodinium, in AUT animals located in 3 contiguous pens suggested that they may have accidentally been cross-faunated before wk 5. The lack of holotrichs in AUT animals throughout the study indicated an incomplete rumen protozoal colonization given the inherent difficulty of holotrichs to become established in the rumen of young ruminants, even under natural milk feeding conditions (Belanche et al., 2010, 2011).

Fermentation end products can be found in the rumen of goat kids as early as the first week of life (Abecia et al., 2014). The presence of fermentation products (mostly VFA) and low pH act as chemical stimuli for the rumen epithelial development (Sander et al., 1959), with butyrate being the most effective followed by propionate and acetate (Baldwin and McLeod, 2000). Our results indicated that the acquisition of microbial populations and the higher solid feed intake triggered fermentation activity in goat kids during the preweaning period, as previously noted in lambs (Abo-Donia et al., 2011; De Barbieri et al., 2015b). As a result, at wk 5 the animals inoculated with fresh rumen fluid had higher $(+47 \%)$ total VFA concentrations than their CTL and AUT counterparts and similar to those observed at wk 7 across treatments $(33 \mathrm{mM})$. This inoculation also enhanced the concentration of butyrate $(+50 \%)$ and ammonia-N $(+26 \%)$ in comparison to CTL and AUT animals at wk 5. Butyrate is transformed into BHB in the rumen wall during absorption; therefore, the higher plasma BHB level observed in animals inoculated with fresh rumen fluid $(+48 \%)$ clearly indicates a higher VFA production and absorption than in CLT and AUT animals before weaning. Several factors could explain this enhanced fermentative activity such as the higher solid feed intake $(+75 \%)$ and the presence of rumen protozoa (Eugène et al., 2004; Khan et al., 2016). A meta-analysis revealed that presence of rumen protozoa, in comparison to defaunated animals, increased rumen OM and NDF digestibility, total VFA, and butyrate concentration as a result of their fibrolytic activity (Newbold et al., 2015). Butyrate has been described as one of the main fermentation products derived from most rumen protozoa (Williams and Coleman, 1992), whereas large protozoa such as Ophryroscolex, Epidinium, Polyplastron, and Eudiplodinium have higher fibrolytic activity (endoglucanase and xylanase) than Entodinium spp. and holotrichs (Takenaka et al., 2004). Thus, the presence of an abundant fibrolytic protozoal population at wk 5 in animals inoculated with fresh rumen fluid could explain the higher rumen fermentation activity than in AUT animals, as previously described in lambs inoculated with fresh (De Barbieri et al., 2015b) or lyophilized rumen fluid (Abo-Donia et al., 2011)

All these observations suggest that during the preweaning phase (wk 5) RFF and RFC animals had a more developed rumen (both microbial and fermentative) than their CTL and AUT counterparts. At weaning (wk 7) animals inoculated with RFC showed higher levels of plasma BHB $(+40 \%)$ and lower levels of glucose $(-7 \%)$ as indicators of a metabolic transition from preruminant to ruminant (Baldwin et al., 2004); however, most differences in terms of rumen fermentation (e.g., ammonia-N and VFA) disappeared across treatments. The similar acetate-to-propionate ratio observed at wk 7 agrees with the similar forage to concentrate ratio consumption across treatments. Possibly the increased feed intake noted in CTL and AUT animals from wk 5 to 7 as compensatory response, and the increase in protozoal numbers in AUT animals, could explain this lack of differences.

\section{Effect of Inoculation After Weaning}

Regarding the third phase of the rumen development (from wk 8), several authors have identified that animals inoculated with fresh rumen fluid had greater DMI than noninoculated animals postweaning (Zhong et al., 2014; De Barbieri et al., 2015b). Our study showed no differences in the postweaning DMI across treatments, but demonstrated that animals inoculated with fresh rumen fluid (particularly with RFC) had increased preference for forage (representing up to $45.5 \%$ of the 
diet), whereas CTL and AUT animals preferred concentrate $(67 \%$ of the diet). Zhong et al. (2014) reported an increase in the apparent digestibility for DM $(+15 \%)$ and NDF $(+35 \%)$ when weaned lambs were inoculated with fresh rumen fluid. Belanche et al. (2019a) found similar results in naturally reared lambs in comparison to those which were artificially reared. This enhanced feed digestibility during postweaning has been associated with higher bacterial (De Barbieri et al., 2015a), protozoal, and anaerobic fungal diversity (Belanche et al., 2019c). Our study showed no differences on the apparent digestibility, possibly because the lower microbial development expected in CTL and AUT animals was compensated by a preferential intake of starter feed as source of easily digestible carbohydrates. This starter preference could also explain their higher levels of total VFA $(+21 \%)$, propionate $(+29 \%)$, and blood glucose $(+4.6 \%)$. On the contrary, the forage preference and high protozoal levels observed in RFF and RFC animals agreed with their higher rumen butyrate $(+35 \%)$ and blood BHB concentrations $(+22 \%)$.

With regard to differences between fresh inocula, De Barbieri et al. (2015b) reported similar short-term increments in rumen protozoa, total VFA, acetate, and bacterial diversity (De Barbieri et al., 2015a) when lambs were inoculated once a week with fresh inocula obtained from donor sheep, which were fed diets rich in either protected fat or coconut oil in comparison with the control group. However, productive responses in this later study were limited given the lack of differences between the 2 types of inocula. Our study showed a similar trend, but the greater concentration of microbes and fermentation products observed in RFC than in RFF inocula would provide further improvements in terms of forage intake, plasma BHB, rumen protozoa, and animal growth during the postweaning period. In this study the daily inoculation was maintained until 4 wk after weaning since it has been reported that although the initial microbial community establishment is affected by early-life interventions, postweaning factors also have a major influence on adult communities and production outcomes (Dill-McFarland et al., 2017). Further taxonomic and functional characterization of the rumen microbiota could elucidate whether RFF or RFC inocula could be used as a rumen microbial programing strategy by favoring the further utilization of forage or concentrate diets later in life (Yáñez-Ruiz et al., 2015). In addition, further studies using a lower inoculation frequency should be performed to make this approach feasible under farm conditions.

With regard to the use of stimulatory compounds in early life, previous studies have shown that inoculation of microbial polysaccharides, cell-free rumen fluid (Muscato et al., 2002), and VFA (mostly butyrate) can stimulate the anatomical (Górka et al., 2018) and microbiological rumen development (O'Hara et al., 2018) with associated positive productive responses. In our study, animals inoculated with autoclaved rumen fluid had higher levels of isobutyrate $(+22 \%)$ and isovalerate $(+41 \%)$ at wk 7 in comparison to other treatments. These isoacids have been described to positively affect bacterial growth, microbial protein synthesis, N retention, and fiber digestion in adult ruminants (Muller, 1987; Liu et al., 2008). However, no substantial improvements of AUT inoculation on rumen fermentation and productivity were observed.

\section{Animal Performance}

In a similar study to this one, De Barbieri et al. (2015a) concluded that rumen fermentation and microbiome composition in lambs can be changed by diet or inoculation with rumen fluid before weaning; however, these changes did not necessarily result in improved performances (De Barbieri et al., 2015b). Our study agrees with the aforementioned because animal performance evaluated up to wk 11 was unaffected by earlylife inoculation. The presence of a complex protozoal population in the rumen of $\mathrm{RFF}$ and RFC animals positively affected the rumen energy metabolism (i.e., higher VFA production and fiber digestion), but this effect could be in part compensated by the negative effect on rumen protein metabolism through bacterial predation by protozoa and the low protozoal contribution to the microbial protein flow in young ruminants (Belanche et al., 2011b). Both factors could result in similar performances across treatments (Belanche et al., 2011a; Newbold et al., 2015). The higher rumen concentration of protein degradation products such as ammonia- $\mathrm{N}(+26 \%)$ at wk 5 and isobutyrate $(+32 \%)$ at wk 9 in animals inoculated with fresh rumen fluid support this hypothesis.

Weaning is a critical phase in ruminant production. Abrupt and early weaning may reduce labor and feeding costs but may cause severe weaning shock, stress, and growth retardation (Khan et al., 2016). Two different weaning programs, based on the weight or the age at weaning, have been described ( $\mathrm{Lu}$ and Potchoiba, 1988). Weaning by weight is considered a low-risk approach because it prevents unhealthy or undernourished kids from being weaned too early. According to Teh et al. (1984), goat kids can be weaned when they reach 3 times their birthweight ( $8.1 \mathrm{~kg}$ in our study). On average this weight was achieved by d 39 of age across treatments (less than $7 \mathrm{wk}$ ), suggesting that weaning could have been implemented earlier. This observation may explain the lack of differences in the cortisol concentration during the postweaning period, suggesting similar 
stress levels across treatments (Moya et al., 2013). Such lack of differences in cortisol levels may also indicate that the daily manipulation of the animals during the inoculation process did not increase the overall stress levels in comparison to absence of inoculation in CTL kids. With regard to weaning by age, Teh et al. (1984) compared the effects of several ages $(4,6,8$, and $10 \mathrm{wk})$ on animal performance, concluding that 8 wk is the optimum for goat kids. Our study showed that weaning at wk 7 was also successful and did not induce weight loss or health problems. Although all kids experienced moderate growth retardation during 1 wk after weaning, inoculation with fresh rumen fluid minimized this weaning shock, leading to 2.2 times higher ADG and 3.7 times higher feed efficiency than CTL and AUT animals during wk 8. Independent of the weaning program considered, weaning should be performed when kids have a sufficient rumen anatomical and fermentative development. It has been suggested that animals should consume at least $30 \mathrm{~g} / \mathrm{d}$ of solid feed before weaning ( $\mathrm{Lu}$ and Potchoiba, 1988). Kids inoculated with fresh rumen fluid met this requirement by wk 5 , whereas 9 extra days were required by their CTL and AUT counterparts. Thus, the higher rumen fermentative development in RFF and RFC kids at wk 5 (e.g., VFA and BHB) suggested that these animals could have succeeded if they went through an early weaning at 5 wk of age.

\section{Cost Analysis}

It has been suggested that early weaning could substantially reduce production costs (Khan et al., 2016). The economic profitability of artificial rearing depends essentially on the cost of milk replacer and the availability of specialized labor (Delgado-Pertíñez et al., 2009). Over this 11-wk study no statistical differences in the total feeding cost across treatments were observed, possibly because all animals were weaned at the same age (wk 7) and the higher milk feeding cost noted in CTL and AUT kids $(+1.45 €)$ was partially compensated by the higher forage feeding cost in their RFF and RFC counterparts $(+0.22 €)$. On average, milk replacer cost was $24 €$ /animal, representing $86 \%$ of the total feeding cost across treatments. In this study the labor-associated costs were not assessed, but results indicated that inoculation with fresh rumen fluid could accelerate the rumen development, making it feasible to perform an early weaning at 6 or even 5 wk of age. This could lead to a decrease in the milk feeding cost by 20 or $35 \%$, respectively, and could contribute to the economic viability of intensive dairy goat farms.

\section{CONCLUSIONS}

This study demonstrated that daily inoculation of young goat kids with fresh rumen fluid from adult animals facilitated early rumen colonization by rumen protozoa, which, together with higher solid feed intake, promoted greater rumen VFA production and absorption during the preweaning period. This intervention minimized the weaning shock as inoculated animals did not experience growth retardation during the postweaning period, making feasible the potential implementation of early weaning strategies. On the contrary, inoculation of autoclaved rumen fluid resulted in negligible (if any) effects on rumen development. Further studies are needed to describe the effect of these strategies on the rumen microbiome, the persistency of the effects under different dietary situations, and the long-term implications on animal productivity.

\section{ACKNOWLEDGMENTS}

This study was funded by the Spanish government through the project AGL2017-86938-R and the Training Program for Academics grant, Madrid, Spain (FPU16/01981). The authors thank Isabel Jimenez and Rosa Serrano (Estación Experimental del Zaidín, Granada, Spain) for their assistance with the animal care and sample analyses. The authors have not stated any conflicts of interest.

\section{REFERENCES}

Abecia, L., E. Ramos-Morales, G. Martínez-Fernandez, A. Arco, A. Martín-García, C. Newbold, and D. Yáñez-Ruiz. 2014. Feeding management in early life influences microbial colonisation and fermentation in the rumen of newborn goat kids. Anim. Prod. Sci. 54:1449-1454. https://doi.org/10.1071/AN14337.

Abo-Donia, F., G. Ibrahim, S. Nadi, and M. Sayah. 2011. Effect of inoculating new born lambs with fresh or lyophilized rumen fluid on rumen activity and lamb performance. J. Am. Sci. 7:409-422.

Arco-Pérez, A., E. Ramos-Morales, D. Yáñez-Ruiz, L. Abecia, and A. Martín-García. 2017. Nutritive evaluation and milk quality of including of tomato or olive by-products silages with sunflower oil in the diet of dairy goats. Anim. Feed Sci. Technol. 232:57-70. https: //doi.org/10.1016/j.anifeedsci.2017.08.008.

Baldwin, R. L., and K. R. McLeod. 2000. Effects of diet forage: concentrate ratio and metabolizable energy intake on isolated rumen epithelial cell metabolism in vitro. J. Anim. Sci. 78:771-783. https: //doi.org/10.2527/2000.783771x.

Baldwin, R. L., K. R. McLeod, J. L. Klotz, and R. N. Heitmann. 2004 Rumen development, intestinal growth and hepatic metabolism in the pre- and postweaning ruminant. J. Dairy Sci. 87:55-65. https: //doi.org/10.3168/jds.S0022-0302(04)70061-2.

Beharka, A. A., T. Nagaraja, J. Morrill, G. Kennedy, and R. Klemm. 1998. Effects of form of the diet on anatomical, microbial, and fermentative development of the rumen of neonatal calves. J. Dairy Sci. 81:1946-1955. https://doi.org/10.3168/jds.S0022 $-0302(98) 75768-6$.

Belanche, A., L. Abecia, G. Holtrop, J. A. Guada, C. Castrillo, G. de la Fuente, and J. Balcells. 2011a. Study of the effect of presence or 
absence of protozoa on rumen fermentation and microbial protein contribution to the chyme. J. Anim. Sci. 89:4163-4174. https://doi .org/10.2527/jas.2010-3703.

Belanche, A., J. Balcells, G. de la Fuente, D. R. Yañez-Ruíz, M. Fondevila, and L. Calleja. 2010. Description of development of rumen ecosystem by PCR assay in milk-fed, weaned and finished lambs in an intensive fattening system. J. Anim. Physiol. Anim. Nutr. (Berl.) 94:648-658. https://doi.org/10.1111/j.1439-0396.2009 .00952.x.

Belanche, A., J. Cooke, E. Jones, H. Worgan, and C. Newbold. 2019a. Short-and long-term effects of conventional and artificial rearing strategies on the health and performance of growing lambs. Animal 13:740-749. https://doi.org/10.1017/S1751731118002100.

Belanche, A., G. de la Fuente, D. R. Yanez-Ruiz, C. J. Newbold, L. Calleja, and J. Balcells. 2011b. Technical note: The persistence of microbial-specific DNA sequences through gastric digestion in lambs and their potential use as microbial markers. J. Anim. Sci. 89:2812-2816. https://doi.org/10.2527/jas.2010-3193.

Belanche, A., E. Jones, I. Parveen, and C. J. Newbold. 2016. A metagenomics approach to evaluate the impact of dietary supplementation with Ascophyllum nodosum or Laminaria digitata on rumen function in Rusitec fermenters. Front. Microbiol. 7:299. https:// doi.org/10.3389/fmicb.2016.00299.

Belanche, A., J. M. Palma-Hidalgo, I. Nejjam, R. Serrano, E. Jiménez, A. I. Martín-García, and D. R. Yáñez-Ruiz. 2019b. In vitro assessment of the factors that determine the activity of the rumen microbiota for further applications as inoculum. J. Sci. Food Agric. 99:163-172. https://doi.org/10.1002/jsfa.9157.

Belanche, A., D. R. Yáñez-Ruiz, A. P. Detheridge, G. W. Griffith, A. H. Kingston-Smith, and C. J. Newbold. 2019c. Maternal vs artificial rearing shapes the rumen microbiome having minor longterm physiological implications. Environ. Microbiol. 21:4360-4377. https://doi.org/10.1111/1462-2920.14801.

Bird, S. H., R. Hegarty, and R. Woodgate. 2010. Modes of transmission of rumen protozoa between mature sheep. Anim. Prod. Sci. 50:414-417. https://doi.org/10.1071/AN09216.

De Barbieri, I., R. Hegarty, C. Silveira, L. Gulino, V. Oddy, R. Gilbert, A. Klieve, and D. Ouwerkerk. 2015a. Programming rumen bacterial communities in newborn Merino lambs. Small Rumin. Res. 129:48-59. https://doi.org/10.1016/j.smallrumres.2015.05.015.

De Barbieri, I., R. Hegarty, C. Silveira, and V. Oddy. 2015b. Positive consequences of maternal diet and post-natal rumen inoculation on rumen function and animal performance of Merino lambs. Small Rumin. Res. 129:37-47. https://doi.org/10.1016/j.smallrumres .2015.05.017.

de Blas, C., G. Mateos, and P. García-Rebollar. 2010. Tablas FEDNA de composición y valor nutritivo de alimentos para la fabricación de piensos compuestos. 3rd ed. FEDNA, Madrid, Spain.

Dehority, B. A. 1993. Laboratory Manual for Classification and Morphology of Ruminal Ciliate Protozoa. CRC Press Inc., Boca Raton, FL.

Delgado-Pertíñez, M., J. Guzmán-Guerrero, Y. Mena, J. Castel, P. González-Redondo, and F. Caravaca. 2009. Influence of kid rearing systems on milk yield, kid growth and cost of Florida dairy goats. Small Rumin. Res. 81:105-111. https://doi.org/10.1016/j .smallrumres.2008.12.007.

De Paula Vieira, A., M. A. G. von Keyserlingk, and D. M. Weary. 2012. Presence of an older weaned companion influences feeding behavior and improves performance of dairy calves before and after weaning from milk. J. Dairy Sci. 95:3218-3224. https://doi .org/10.3168/jds.2011-4821.

Dill-McFarland, K. A., J. D. Breaker, and G. Suen. 2017. Microbial succession in the gastrointestinal tract of dairy cows from 2 weeks to first lactation. Sci. Rep. 7:1-12.

Eugène, M., H. Archimède, and D. Sauvant. 2004. Quantitative metaanalysis on the effects of defaunation of the rumen on growth, intake and digestion in ruminants. Livest. Prod. Sci. 85:81-97. https: //doi.org/10.1016/S0301-6226(03)00117-9.

Ewan, R., E. Hatfield, and U. Garrigus. 1958. The effect of certain inoculations on the utilization of urea or biuret by growing lambs. J. Anim. Sci. 17:298-303. https://doi.org/10.2527/jas1958.172298x.
Górka, P., Z. Kowalski, R. Zabielski, and P. Guilloteau. 2018. Invited review: Use of butyrate to promote gastrointestinal tract development in calves. J. Dairy Sci. 101:4785-4800. https://doi.org/10 $.3168 /$ jds.2017-14086.

Heinrichs, A. 2005. Rumen development in the dairy calf. Adv. Dairy Technol. 17:179-187.

Hidiroglou, M. 1979. Manganese in ruminant nutrition. Can. J. Anim. Sci. 59:217-236. https://doi.org/10.4141/cjas79-028.

Khan, M., A. Bach, D. Weary, and M. von Keyserlingk. 2016. Transitioning from milk to solid feed in dairy heifers. J. Dairy Sci. 99:885-902. https://doi.org/10.3168/jds.2015-9975.

Kim, S. W., J. F. Less, L. Wang, T. Yan, V. Kiron, S. J. Kaushik, and X. G. Lei. 2019. Meeting global feed protein demand: Challenge, opportunity, and strategy. Annu. Rev. Anim. Biosci. 7:221-243. https://doi.org/10.1146/annurev-animal-030117-014838.

Lane, M. A., R. L. Baldwin, and B. W. Jesse. 2000. Sheep rumen metabolic development in response to age and dietary treatments. J. Anim. Sci. 78:1990-1996. https://doi.org/10.2527/2000.7871990x.

Li, R., Z. Teng, C. Lang, H. Zhou, W. Zhong, Z. Ban, X. Yan, H. Yang, M. H. Farouk, and Y. Lou. 2019. Effect of different forageto-concentrate ratios on ruminal bacterial structure and real-time methane production in sheep. PLoS One 14:e0214777. https://doi .org/10.1371/journal.pone.0214777.

Liu, Q., C. Wang, Y. Huang, K. Dong, H. Wang, and W. Yang. 2008. Effects of isobutyrate on rumen fermentation, urinary excretion of purine derivatives and digestibility in steers. Arch. Anim. Nutr. 62:377-388. https://doi.org/10.1080/17450390802327761.

Lu, C. D., and M. Potchoiba. 1988. Milk feeding and weaning of goat kids - A review. Small Rumin. Res. 1:105-112. https://doi.org/10 .1016/0921-4488(88)90025-9.

McAllister, T., K. Beauchemin, A. Alazzeh, J. Baah, R. Teather, and K. Stanford. 2011. The use of direct fed microbials to mitigate pathogens and enhance production in cattle. Can. J. Anim. Sci. 91:193-211. https://doi.org/10.4141/cjas10047.

Meale, S. J., S. Li, P. Azevedo, H. Derakhshani, J. C. Plaizier, E. Khafipour, and M. A. Steele. 2016. Development of ruminal and fecal microbiomes are affected by weaning but not weaning strategy in dairy calves. Front. Microbiol. 7:582. https://doi.org/10 .3389/fmicb.2016.00582.

Moya, D., K. Schwartzkopf-Genswein, and D. Veira. 2013. Standardization of a non-invasive methodology to measure cortisol in hair of beef cattle. Livest. Sci. 158:138-144. https://doi.org/10.1016/j livsci.2013.10.007.

Muller, L. D. 1987. Branched chain fatty acids (Isoacids) and valeric acid for ruminants. Prof. Anim. Sci. 3:9-12. https://doi.org/10 .15232/S1080-7446(15)32370-6.

Muscato, T., L. Tedeschi, and J. Russell. 2002. The effect of ruminal fluid preparations on the growth and health of newborn, milk-fed dairy calves. J. Dairy Sci. 85:648-656. https://doi.org/10.3168/jds .S0022-0302(02)74119-2.

Newbold, C. J., G. de la Fuente, A. Belanche, E. Ramos-Morales, and N. McEwan. 2015. The role of ciliate protozoa in the rumen. Front. Microbiol. 6:1313. https://doi.org/10.3389/fmicb.2015.01313.

O'Hara, E., A. Kelly, M. S. McCabe, D. A. Kenny, L. L. Guan, and S. M. Waters. 2018. Effect of a butyrate-fortified milk replacer on gastrointestinal microbiota and products of fermentation in artificially reared dairy calves at weaning. Sci. Rep. 8:14901. https:// doi.org/10.1038/s41598-018-33122-6.

Ramos-Morales, E., A. Arco-Pérez, A. I. Martín-García, D. R. YáñezRuiz, P. Frutos, and G. Hervás. 2014. Use of stomach tubing as an alternative to rumen cannulation to study ruminal fermentation and microbiota in sheep and goats. Anim. Feed Sci. Technol. 198:57-66.

Rodríguez, C., and A. Rodríguez. 2011. Effect on weight gain, rumen bioactivity and rumen $\mathrm{pH}$ in weaned sheep using fresh rumen fluid from cattle. Rev. Mvz Cordoba 16:2692-2700.

Sander, E., R. Warner, H. Harrison, and J. Loosli. 1959. The stimulatory effect of sodium butyrate and sodium propionate on the development of rumen mucosa in the young calf. J. Dairy Sci. 42:1600-1605. https://doi.org/10.3168/jds.S0022-0302(59)90772 -6 . 
Takenaka, A., K. Tajima, M. Mitsumori, and H. Kajikawa. 2004. Fiber digestion by rumen ciliate protozoa. Microbes Environ. 19:203210. https://doi.org/10.1264/jsme2.19.203.

Teh, T., M. Potchoiba, E. Escobar, and C. Lu. 1984. Weaning methods of goat kids. J. Dairy Sci. 67:137.

Uyeno, Y., S. Shigemori, and T. Shimosato. 2015. Effect of probiotics/ prebiotics on cattle health and productivity. Microbes Environ. 30:126-132. https://doi.org/10.1264/jsme2.ME14176.

Waymack, L. 1976. Effect of feeding lyophilized rumen contents on adaptation to urea diet by lambs. J. Anim. Sci. 43:712-714. https: //doi.org/10.2527/jas1976.433712x.

Weatherburn, M. W. 1967. Phenol-hypochlorite reaction for determination of ammonium. Anal. Chem. 39:971-974. https://doi.org/10 .1021/ac60252a045.

Weimer, P. J. 2015. Redundancy, resilience, and host specificity of the ruminal microbiota: Implications for engineering improved ruminal fermentations. Front. Microbiol. 6:296. https://doi.org/10.3389/ fmicb.2015.00296.

Williams, A. G., and G. S. Coleman. 1992. The Rumen Protozoa. Springer-Verlag, New York, NY.

Yáñez-Ruiz, D. R., L. Abecia, and C. J. Newbold. 2015. Manipulating rumen microbiome and fermentation through interventions during early life: A review. Front. Microbiol. 6:1133. https://doi.org/10 .3389/fmicb.2015.01133.

Yoon, I., and M. Stern. 1995. Influence of direct-fed microbials on ruminal microbial fermentation and performance of ruminants: A review. Asian-australas. J. Anim. Sci. 8:533-555. https://doi.org/ 10.5713 /ajas.1995.553

Zhong, R., H. Sun, G. Li, H. Liu, and D. Zhou. 2014. Effects of inoculation with rumen fluid on nutrient digestibility, growth performance and rumen fermentation of early weaned lambs. Livest. Sci. 162:154-158. https://doi.org/10.1016/j.livsci.2013.12.021.

\section{ORCIDS}

A. Belanche 주 https://orcid.org/0000-0001-5880-6021

J. M. Palma-Hidalgo @ https://orcid.org/0000-0002-6237-1822

A. I. Martín-García ำ https://orcid.org/0000-0002-5366-3938

D. R. Yáñez-Ruiz @ https://orcid.org/0000-0003-4397-3905 